\title{
ATIVIDADE DA LACTATO DESIDROGENASE (LDH) SÉRICA EM CÃES SUBMETIDOS À OXIGENAÇÃO EXTRACORPÓREA POR MEMBRANA (ECMO) POR UM PERÍODO DE TRÊS HORAS
}

\author{
Felipp Silveira Ferreira ${ }^{1}$, Lara Lages Silveira ${ }^{2}$, Alessandra Castello Costa ${ }^{2}$, Daniela

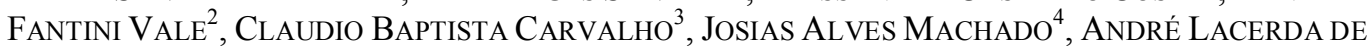 \\ ABREU OLIVEIRA ${ }^{3}$ \\ ${ }^{1}$ Doutorando em Ciência Animal pela Universidade Estadual do Norte Fluminense, Campos dos Goytacazes - \\ felipp@gigalink.com.br \\ ${ }^{2}$ Pós-graduandos da Universidade Estadual do Norte Fluminense \\ ${ }^{3}$ Professor Doutor da Universidade Estadual do Norte Fluminense \\ ${ }^{4}$ Biólogo da Prefeitura Municipal de Carapebus
}

RESUMO

\begin{abstract}
A oxigenação extracorpórea por membrana (ECMO) é uma técnica de suporte cardiopulmonar prolongado, que objetiva auxiliar os pulmões e/ou o coração quando estes não respondem aos tratamentos convencionais. Este estudo avaliou a atividade da lactato desidrogenase (LDH) sérica de cinco cães sem raça definida (SRD) submetidos à ECMO por um período de três horas. Sob pressão expiratória positiva final (PEEP) de $10 \mathrm{mmHg}$, ventilação controlada e $\mathrm{FiO}_{2}$ de $21 \%$, a ECMO foi realizada por canulação femoral (artéria e veia), em um desvio artério-
\end{abstract}

venoso (AV). A atividade da LDH foi mensurada a cada trinta minutos, por três horas e os resultados analisados estatisticamente com os testes de ANOVA e Tukey, com $=5 \%$. O resultado apontou um aumento sérico na atividade da LDH, caracterizando uma lesão muscular ao longo do procedimento, em virtude de uma resposta fisiológica semelhante àquela causada por um choque hipovolêmico. Conclui-se que a ECMO é uma técnica viável de suporte, mas necessita de adaptações para uso clínico em cães.

PALAVRAS-CHAVE: cães; ECMO; lactato desidrogenase.

\section{SERUM LACTATE DEHYDROGENASE (LDH) ACTIVITY IN DOGS SUBMITTED TO EXTRACORPOREAL MEMBRANE OXYGENATION (ECMO) FOR THREE HOURS}

\section{ABSTRACT}

The extracorporeal membrane oxygenation (ECMO) is a prolonged cardiopulmonary support technique, which aims to help the lungs and the heart when they do not respond to conventional non-invasive treatments. This research was carried out to determine the behavior of lactate dehydrogenase (LDH) of five mongrel dogs undergoing ECMO for three hours. Under controlled ventilation, positive end-expiratory pressure (PEEP) at $10 \mathrm{mmHg}$ and $\mathrm{FiO}_{2}$ at $21 \%$, the animals were submitted to femoral cannulation for ECMO (artery and vein), by the arterial-venous (AV) deviation. The LDH was measured and evaluated every thirty minutes for an uninterrupted period of three hours. The results were tabulated and statistically analyzed with ANOVA and Tukey tests, with $p<0.05$. The results showed an increase of serum LDH, featuring a muscle injury during the procedure due to a physiological response, similar to that caused by a hypovolemic shock. We concluded that ECMO is a viable technique for prolonged ventilatory support, but it needs some adjustments for clinical use in dogs.

KEYWORDS: dogs: ECMO; lactate dehydrogenase. 


\section{INTRODUÇÃO}

A oxigenação extracorpórea por membrana (ECMO) é uma técnica de suporte cardiopulmonar prolongado, com o objetivo de auxiliar o pulmão e/ou o coração, na maioria das vezes de neonatos e crianças, quando estes apresentam processos de falência não responsiva aos tratamentos convencionais não-invasivos (BARTLETT et al., 1974, WEBER et al., 1990, MOSCARDINI et al., 2002, PEEK et al., 2006, TAMESUE et al., 2006, KAHN et al., 2007). Entretanto, essa técnica deve ser realizada apenas nos casos em que a insuficiência respiratória for causada por uma enfermidade potencialmente reversível (SOUZA \& ELIAS, 2006a).

Em humanos, é recomendada para pacientes com a respiração prejudicada de forma aguda e para o tratamento de pacientes com disfunção miocárdica severa e reversiva, como cardiomiopatias ou choque cardiogênico pósoperatório (LIN et al., 2006; PEEK et al., 2006). Em recém-nascidos, a técnica é empregada em vários casos, tais como na Síndrome da Aspiração do Mecônio, hérnias diafragmáticas congênitas, hipertensão pulmonar persistente do recém-nascido e pneumonias e sepses (VORST et al., 2006). A ECMO também deve ser considerada em casos refratários à terapêutica farmacológica para a Hipertensão Pulmonar (HP) em crianças. No entanto, o índice de sucesso desta modalidade terapêutica em pós-operatório de cirurgia cardíaca com HP é menor do que na HP primária do recémnascido (HOROWITZ, 2002).

Em adultos, possíveis indicações da ECMO são doenças respiratórias agudas, como a síndrome da angústia respiratória, hipóxicas ou hipercárbicas, insuficiência cardíaca direita aguda e insuficiência cardíaca global aguda, porém sempre nos casos em que a reversibilidade for esperada. A ECMO também é utilizada em casos de transplantes de pulmões e/ou coração (KAHN et al., 2007). Neste caso, segundo HOROWITZ (2002), com bastante utilidade quando o suporte inotrópico não funciona no pós-cirúrgico; assim, a ECMO tem sido utilizada nessas situações com o objetivo de manter a perfusão tecidual, minimizar os gastos energéticos, maximizar a recuperação miocárdica e como ponte para transplante. Ainda de acordo com HOROWITZ (2002), o índice de sucesso varia conforme a instituição, mas é consenso que melhora a sobrevida em crianças com síndrome de baixo débito cardíaco e hipertensão pulmonar severa.
No Brasil, em Medicina Veterinária, a ECMO vem sendo inserida gradativamente por COSTA et al. (2009) e FERREIRA et al. (2010) em cães com insuficiência respiratória aguda potencialmente reversível e em estudos experimentais.

Para a realização desse procedimento, existem três tipos de desvio cardiopulmonar. Dentre eles, o desvio artério-venoso é o menos utilizado nos procedimentos de assistência ventilatória. Ele ocorre através da retirada do sangue pela artéria femoral, retornando através da veia jugular interna, após a hematose. Seu uso é considerado para prematuros, como uma alternativa quando os demais tipos não são aplicáveis. É importante que haja um correto balanço dos fluxos; o mesmo volume de sangue removido deve retornar ao paciente, de modo que a hemodinâmica do paciente mantenha-se estável (SOUZA \& ELIAS, 2006a).

A ECMO é um procedimento não fisiológico. Em todos os pacientes submetidos à circulação extracorpórea, principalmente os pacientes pediátricos, os efeitos da hipotermia, da distribuição irregular do fluxo sanguíneo aos tecidos, da hemodiluição, dos desvios do equilíbrio ácido-base, das microembolias e da resposta inflamatória sistêmica do organismo continuam representando um desafio de grandes proporções (SOUZA \& ELIAS, 2006b).

Segundo BARTLETT et al. (1974), a aplicação clínica da ECMO é aparentemente viável, porém é cada vez mais importante estabelecer as respostas orgânicas frente ao procedimento. De acordo com o autor, corroborado por SOUSA \& ELIAS (2006a), a hipotensão causada pelo procedimento pode causar a redução do fluxo sanguíneo para as extremidades, o que resultaria em uma lesão tecidual.

Assim, como descrito por DUNCAN \& PRASSE (1986), MEYER et al. (1995) e KANEKO et al. (1997), a aferição da LDH é uma das formas de avaliação da função muscular em medicina veterinária, podendo revelar possíveis lesões teciduais e inflamatórias, embora não se configure como um marcador de alta especificidade, embora apresente relativa significância.

Por esse motivo, realizou-se esta pesquisa com a finalidade de avaliar a atividade da enzima Lactato Desidrogenase sérica (LDH) em modelos caninos de cães submetidos à ECMO, com o intuito de estabelecer seus parâmetros e tornar o procedimento mais seguro, reduzindo a incidência das relativas complicações. 


\section{MATERIAL E MÉTODOS}

Este trabalho foi realizado no centro cirúrgico do Hospital Veterinário da Universidade Estadual do Norte Fluminense Darcy Ribeiro (UENF).

Para a presente pesquisa, foram utilizados cinco cães SRD, sem restrição de sexo e idade, com peso corporal entre 10 e 15 quilos. Todos os animais foram cedidos pelo Centro de Controle de Zoonoses de Campos dos Goytacazes, RJ. Para a padronização dos animais, eles foram identificados como A1 (animal 1), A2 (animal 2), A3 (animal 3), A4 (animal 4) e A5 (animal 5). Os animais foram admitidos no Hospital Veterinário da UENF, onde foram realizados exames clínicos e laboratoriais (hemograma e bioquímica sérica), a fim de detectar quaisquer alterações orgânicas. Os animais enfermos foram descartados do experimento.

Posteriormente, foram alocados em gaiolas apropriadas, recebendo alimentação especializada, água ad libitum, limpeza e banhos de sol, objetivando seu bem-estar.

Os animais utilizados foram anestesiados a partir do seguinte protocolo: $0,1 \mathrm{mg} \cdot \mathrm{kg}^{-1}$ de Acepromazina ${ }^{1}$ por via intravenosa (IV), seguido de $2 \mathrm{mg} \cdot \mathrm{kg}^{-1}$ de Cetamina ${ }^{2} \mathrm{IV}$, posteriormente $7 \mathrm{mg} \cdot \mathrm{kg}^{-1}$ de Lidocaína $^{3}$ por via epidural. A manutenção anestésica foi feita com Isoflurano ${ }^{4} 2,5 \%$. A anticoagulação foi realizada com Heparina ${ }^{5}$, na dose única de $4 \mathrm{mg} \cdot \mathrm{kg}^{-1} \mathrm{IV}$.

Depois de anestesiados e intubados, os animais foram colocados em decúbito dorsal e submetidos à ECMO pela utilização da técnica de desvio artério-venoso, com a retirada do sangue pela artéria femoral e a reinfusão pela veia femoral. O sangue seguiu o seguinte trajeto: artéria femoral, tubo conector, oxigenador de membranas e, após a remoção do $\mathrm{CO}_{2}$ e o recebimento de $\mathrm{O}_{2}$, o sangue fluiu de volta para o organismo através de outro tubo conector, chegando à veia femoral. Os animais permaneceram sob ECMO durante 180 minutos, com o Oxigenador de Membranas ${ }^{6}$.

Para a manutenção sob ECMO, os animais receberam um suporte ventilatório mínimo, com

\footnotetext{
1 Acepran ${ }^{\circledR}$, Univet S/A, Rua Clímaco Barbosa 700, Cambuci, São Paulo-SP.

2 Ketamina ${ }^{\circledR}$, Agener União Química Farmacêutica Nacional, Rua Cel. Luiz Tenório de Brito 90, Embu-Guaçu, São Paulo-SP.

${ }^{3}$ Lidovet®, Laboratório Bravet LTDA, Rua Visconde de Santa Cruz 276, Engenho Novo, Rio de Janeiro-RJ.

4 Isoforine ${ }^{\circledR}$, Cristália Produtos Químicos Farmacêuticos Ltda, Rod. Itapira-Lindóia Km 14, Itapira-SP

5 Heparin ${ }^{5}$, Cristália Produtos Químicos Farmacêuticos Ltda, Rod. Itapira-Lindóia Km 14, Itapira-SP.

${ }^{6}$ Kids D100®, Sorin Group Italia, Via Statale 12 Nord 86, Mirandola, Modena, Itália.
}

pressão expiratória positiva final (PEEP) de $10 \mathrm{~cm}$ $\mathrm{H}_{2} \mathrm{O}$ e $\mathrm{FiO}_{2}$ de $21 \%$, concomitantemente à terapia de suporte promovida pela ECMO, objetivando evitar atelectasias, e as funções dos pulmões foram substituídas apenas parcialmente pelo órgão artificial.

Os animais receberam, ainda, Dopamina ${ }^{7}$, na dose de $5 \mu \mathrm{g} / \mathrm{kg} /$ minuto, em infusão contínua, como suporte pressórico e inotrópico.

As amostras de sangue venoso foram coletadas por seringas de $3 \mathrm{~mL}$ conectadas a um cateter longo localizado na veia jugular, o qual foi guiado em direção ao átrio direito. $\mathrm{O}$ sangue coletado foi repassado em frascos siliconizados sem anticoagulante. $\mathrm{O}$ intervalo entre as coletas foi estipulado em 30 minutos, durante três horas, totalizando sete amostras por animal durante a ECMO. Também foi realizada a coleta de uma amostra de sangue do animal antes da ECMO, para controle, totalizando oito tempos de coleta durante o período total de experimentação.

As amostras sanguíneas foram centrifugadas (1500g por 5 minutos), em tempo não superior a uma hora, e o produto sobrenadante foi transformado em aliquota após a coleta em volume mínimo de $500 \mu \mathrm{L}$. Tais amostras foram acondicionadas em microtubos tipo "Eppendorf" e mantidas sob refrigeração em "freezer", à temperatura de $-20^{\circ} \mathrm{C}$, para posterior realização de análises bioquímicas da $\mathrm{LDH}$, em equipamento espectrofotométrico ${ }^{8}$ e kits comerciais específicos ${ }^{9}$.

Após o estudo, os animais foram submetidos à eutanásia com administração de tiopental sódico ${ }^{10}$, na dose de $40 \mathrm{mg} \cdot \mathrm{kg}^{-1} \mathrm{IV}$, e cloreto de potássio ${ }^{11}$, na dose de $100 \mathrm{mg} \cdot \mathrm{kg}^{-1} \mathrm{IV}$, respeitando-se os princípios éticos do uso de animais de experimentação.

O presente trabalho foi licenciado pelo Comitê de Ética para Uso de Animais da Universidade Estadual do Norte Fluminense Darcy Ribeiro, sob o registro CEUA-UENF 038/2008.

Os resultados foram anotados em fichas individuais (oito para cada animal) e as diferenças entre os momentos foram analisadas com programa estatístico GraphPad Prism versão 4.03 for Windows (GraphPad Software), através dos testes One-way ANOVA (Friedman e Kruskal-Wallis), com pós teste

\footnotetext{
${ }^{7}$ Cloridrato de Dopamina ${ }^{\circledR}$, Laboratório Teuto Brasileiro S/A, VP 7-D módulo 11 - Quadra 13, Anápolis-GO.

${ }^{8}$ Labmax Plenno®, Labtest Diagnóstica, Av. Paulo Ferreira da Costa 600, Lagoa Santa-MG.

${ }^{9}$ LDH Liquiform Ref. 86®, Labtest Diagnóstica, Av. Paulo Ferreira da Costa 600, Lagoa Santa-MG

10 Thiopentax ${ }^{\circledR}$, Cristália Produtos Químicos Farmacêuticos Ltda, Rod. Itapira-Lindóia Km 14, Itapira-SP.

11 Cloreto de Potássio®, Equiplex Indústria Farmacêutica, Rua Thubérgia Qd. K No 233, Aparecida de Goiânia-GO.
} 
de comparações múltiplas de Tukey, com p<0,05.

\section{RESULTADOS E DISCUSSÃO}

Na Figura 1 está representada a avaliação da atividade da enzima lactato desidrogenase (LDH) do grupo de animais submetidos à ECMO. Pela análise da linha de tendência apresentada, conclui-se que a LDH apresentou uma tendência de aumento na sua atividade ao longo das três horas de ensaio.

Os dados da análise estatística, por ANOVA $(\mathrm{p}<0,05)$, apesentaram uma não significância entre os momentos analisados. Entretanto, é relevante destacar a seguinte situação: no momento 0 (M0), o gráfico (Figura 1) apresenta um destaque para o ponto referente ao Animal 1 (A1) do grupo, o qual apresentou um comportamento que difere do restante do grupo, com alta atividade enzimática, contrapondo os valores mais baixos observados nos demais animais. Em uma análise inicial, descarta-se a possibilidade de uma alteração induzida pela ECMO, uma vez que o animal apenas esteve sob ECMO a partir do momento 1 (M1). Enquanto os demais animais apresentavam valores próximos de 200U/L, este animal em destaque apresentava um valor superior a $600 \mathrm{U} / \mathrm{L}$ por motivos não elucidados. Cabe relatar, ainda, que este animal não manteve esse nível elevado nos momentos posteriores, excluindo-se a possibilidade de que ele possa ter apresentado níveis séricos de LDH que possam ter comprometido os momentos posteriores de avaliação.

Outro fato interessante a ser destacado é que mesmo não apresentando diferenças estatisticamente significativas entre si, todos os valores médios da LDH a partir de M3, ou seja, 1 hora de ECMO, encontravam-se além dos valores de referência para cães $(63,0-270,0 \mathrm{U} / \mathrm{L}$, segundo KANEKO et al. (1997)). Esse fato é semelhante ao descrito inicialmente por BARTLETT et al. (1974), em seu ensaio de oxigenação extracorpórea com ovelhas. Destaca-se o fato que também em M0 os valores estão acima do referencial para a espécie; contudo, isso se deve ao comportamento aberrante do A1, o qual, neste caso, foi o responsável direto pela elevação da média neste momento. A título de informação, excluindo-se o valor do animal (A1) no momento (M0), a nova média seria em torno de 152,5 U/L, dentro dos parâmetros de normalidade para cães.

Com base no descrito por DUNCAN \& PRASSE (1986) e MEYER et al. (1995) sobre a LDH ser uma enzima localizada em diversos tecidos, sobretudo musculares, somado aos achados experimentais de BARTLETT et al. (1974) sobre a LDH e aos eventos de hipoperfusão periférica desencadeados pela ECMO descritos por SOUSA \& ELIAS (2006a), pode-se concluir que a ECMO causou uma lesão muscular considerável a partir de uma hora de procedimento. Esse resultado é semelhante ao descrito por MARTINS et al. (2002), que, por sua vez, atribuíram a variação da $\mathrm{LDH}$, durante seu experimento de circulação extracorpórea em bezerros, não somente ao estresse causado pelo procedimento, mas também à hemólise.

A descrição dos valores médios da $\mathrm{LDH}$ por tempo de ECMO é mostrada no Quadro 1.

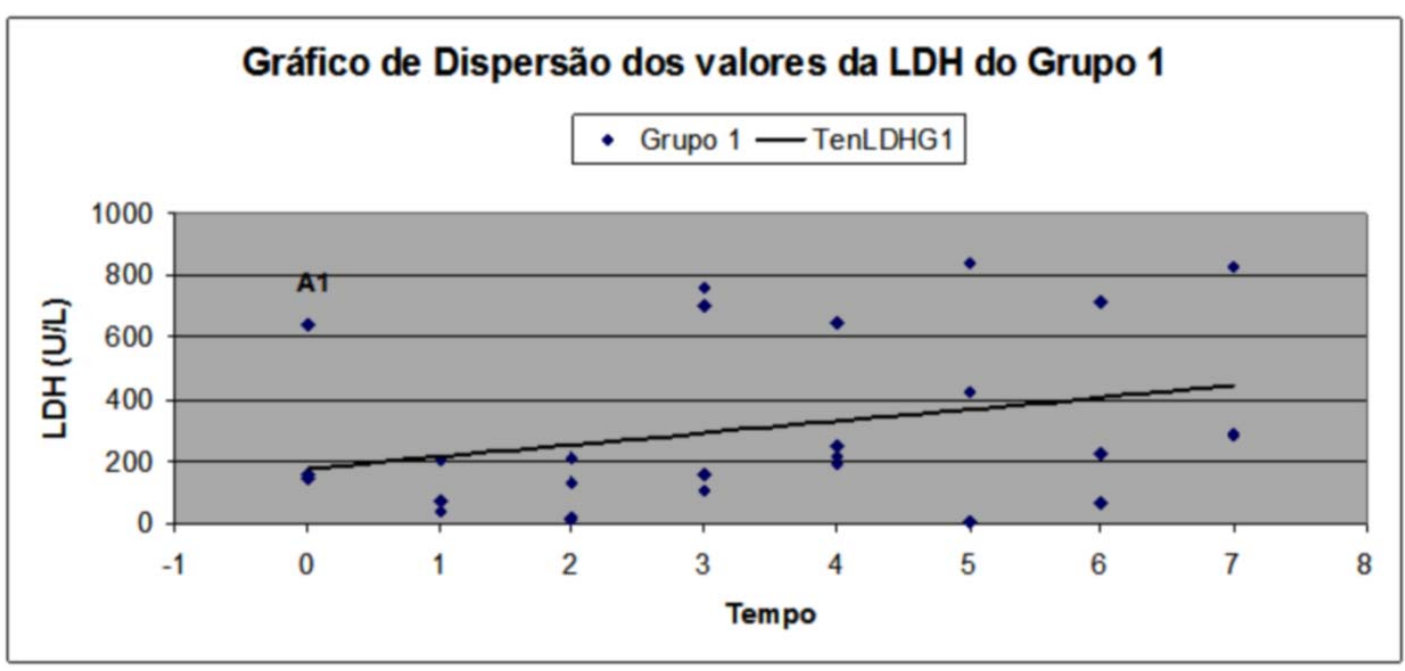

Fig.1. Dispersão e comportamento da enzima Lactato desidrogenase (LDH), em U/L, de cães durante um período de três horas de ECMO. Como descrito no texto, destaca-se um comportamento fora do padrão no ponto A1. Embora cada ponto represente um animal, não se observam cinco pontos por momento, pois alguns estão sobrepostos ou sua ausência efetiva indica o óbito do animal durante o procedimento. TenLDHG1: Linha de tendência da atividade da LDH sérica dos animais estudados. 
Quadro 1. Médias e análise estatística da atividade da enzima lactato desidrogenase (LDH) durante o período de três horas de ECMO em oito tempos (M0 a M7)

\begin{tabular}{|c|c|c|c|c|c|c|c|c|}
\hline Tempos & M0 & M1 & M2 & M3 & M4 & M5 & M6 & M7 \\
\hline $\mathrm{LDH}(\mathrm{U} / \mathrm{L})$ & 315,3 & 107,0 & 93,5 & 431,5 & 328,5 & 422,7 & 334,7 & 468,0 \\
\hline Análise estatística & \multicolumn{8}{|c|}{ ANOVA, significativo $\alpha=5 \%$; Tukey Signficativo $\alpha=5 \%$} \\
\hline Referência $(\mathrm{U} / \mathrm{L})^{\mathrm{a}}$ & \multicolumn{8}{|c|}{$63,0-270,0$} \\
\hline
\end{tabular}

${ }^{\mathrm{a}}$ Kaneko et al. (1997).

Esses achados corroboram as informações de GUYTON \& HALL (2006) e SOUSA \& ELIAS (2006a). De acordo com os autores, em resposta à circulação extracorpórea, ou à hipotensão, o organismo tende à ativação de barorreceptores, que, por sua vez, desencadeiam uma série de reações visando o restabelecimento da pressão arterial. Dentre esses mecanismos, a ativação do sistema nervoso simpático resulta em um aumento da resistência vascular periférica. Isso torna a velocidade do fluxo sanguíneo para algumas regiões reduzida, o que resulta em déficit no aporte de oxigênio e nutrientes necessários à manutenção das funções fisiológicas do tecido. Paralelamente a tal fato, ocorre uma acessória redistribuição do fluxo de sangue para os órgãos mais nobres. Em detrimento disso, outros órgãos, como rins, fígado e as massas musculares recebem fluxos de sangue insuficientes para as suas necessidades. Em função dessa condição, produz-se um quadro propício ao dano muscular, caracterizado pelos aumentos séricos da LDH no presente trabalho.

Acredita-se que, frente aos resultados apresentados, a ECMO constitua um elemento importante e viável para o suporte respiratório prolongado; entretanto, necessita de algum aperfeiçoamento ou adaptação para utilização na prática clínica em medicina veterinária.

\section{CONCLUSÕES}

A ECMO comportou-se como uma técnica viável de suporte respiratório prolongado, porém, através da análise da atividade sérica da $\mathrm{LDH}$, conclui-se que ela produz danos musculares em cães após três horas de procedimento, necessitando, ainda, de mais estudos para que se possa, definitivamente, reduzir os riscos associados à técnica em questão.

\section{AGRADECIMENTOS}

Ao Laboratório Terceiro Milênio (Pedra
Verde) pela disponibilização do equipamento espectrofotômétrico para realização dos ensaios bioquímicos.

\section{REFERÊNCIAS}

BARTLETT, R.H., FONG, S.W., BURNS, N.E.; GAZZANIGA, A.B. Prolonged Partial Venoarterial Bypass: Physiologic, Biochemical, and Hematologic Responses. Annals of Surgery, v.180, n.6, p.850-856, 1974.

COSTA, A.C., OLIVEIRA, A.L.O., ANTUNES, F., ABÍLIO, E.J., CARVALHO, E.C.Q., ALBERNAZ, A.P., SANTOS, C.L., FERREIRA, F.S., SILVEIRA, L.L., SALIBA, R., CARVALHO, C.B., MACHADO, J.A. Oxigenação extracorpórea por membrana (ECMO) sem auxílio circulatório em canino. MEDVEP, v.19, p.293300,2009

DUNCAN, J.R.; PRASSE, K.W. Veterinary laboratory medicine, clinical pathology. 2.ed. Ames: Iowa State University, 1986. 285p.

FERREIRA, F.S., SILVEIRA, L.L., COSTA, A.C., ALBERNAZ, A.P., CARVALHO, C.B., OLIVEIRA, A.L.A. Estudo do comportamento da Creatino Quinase (CK) e Creatino Quinase-MB (CK-MB) sérica de cães submetidos à Oxigenação por membrana extracorpórea (ECMO) durante um período de três horas. Ciência Animal Brasileira, v. 11, n. 3, p.705-712, 2010.

GUYTON, A.C., HALL, J.E. Textbook of Medical Physiology. Philadelphia: Elsevier Saunders, p.161-215, 2006.

HOROWITZ, E.S.K. Pós-operatório de cirurgia cardíaca: síndrome de baixo débito e crise de hipertensão pulmonar. Revista Médica do Instituto de Cardiologia do Rio Grande do Sul, v.2, n.2, p.115-120, 2002.

KAHN, J., MULLER, H., MARTE, W., REHAK, P., WASLER, A., PRENNER, G., TSCHELIESSNIGG, K. Establishing extracorporeal membrane oxygenation in a university clinic: case series. Journal of Cardiothoracic and Vascular Anesthesia, v.21, n.3, p.384-387, 2007.

KANEKO, J.J., HARVEY, J.W., Bruss, M.L. Clinical 
Biochemestry of Domestic Animals. Califórnia: Academic Press, 1997. p.303-325; 462-495.

LIN, C., CHEN, Y., TSAI, F., TIAN, Y., JENQ, C., FANG, J., YANG, C. RIFLE Classification Is Predictive Of Short-Term Prognosis In Critically Ill Patients With Acute Renal Failure Supported By Extracorporeal Membrane Oxygenation. Nephrology Dialysis Transplantation, v.21, p.2867-2873, 2006.

MARTINS, A.M.S., NOGAROTO, S., PICCINI, B., TOFFANO, R., VAMPEL, F., BISCEGLI, J.F. Avaliação de oxigenador de membrana durante teste in vivo. Revista Latinoamericana de Tecnologia Extracorpórea, v.9, n.3, 2002 .

MEYER, D.J.; COLES, E.H., RICH, L.J. Medicina de Laboratório Veterinária - Interpretação e Diagnóstico. São Paulo: Editora Roca. 1995. 308p.

MOSCARDINI, A.C., GODOY, M.F., BRAILE, D.M., GODOY, J.M.P., SOARES, M.J., BRANDI, A.C., RAMIN, S.L. Oxigenação extracorpórea por membrana e alterações hematológicas em estudo experimental. Revista Brasileira de Hematologia e Hemoterapia, v.24, n.2, p.97-104, 2002.

PEEK, G.J., CLEMENS, F., ELBOURNE, D., FIRMIN, R.K., HARDY, P., HIBBERT, C., KILLER, H., MUGFORD, M., THALANANY, M., TIRUVOIPATI, R., TRUESDALE, A., WILSON, A. CESAR: conventional ventilatory support vs extracorporeal membrane oxygenation for severe adult respiratory failure. BMC Health Services Research, v.6, n.163, p.1$13,2006$.

SOUZA, M.H.L., ELIAS, D.O. Fundamentos da Circulação Extracorpórea. Rio de Janeiro: Centro Editorial Alfa, 2006a. p.377-406; 755-766.

SOUZA, M.H.L., ELIAS, D.O. Perfusão Pediátrica. Perfusion Line, Boletim Informativo - Centro De Estudos Alfa Rio, v.4, n.8, p.5-6, 2006 b.

TAMESUE, K., ICHIBA, S., NAWA, S., SHIMIZU, N. An Experimental Study On Pumpless Extracorporeal Membrane Oxigenation (Ecmo) Support In A Canine Model. Acta Medica Okayama, v.60, n.3, p.167-172, 2006.

VORST, M.M.J.; WILDSCHUT, E., HOUMES, R.J., GISCHLER, S.J., HOLTHE, J.E.K., BURGGRAF, J., HEIJDEN, A., TIBBOEL, D. Evaluation of furosemide regimens in neonates treated with extracorporeal membrane oxygenation. Critical Care, v.10, n.6, p.1-8, 2006.

WEBER, T.R., CONNORS, R.H., TRACY JR, T.F., BAILEY, P.V., STEPHENS, C., KEENAN, W. Prognostic determinants in extracorporeal membrane oxygenation for respiratory failure in newborns. The Annals of Thoracic Surgery, v.50, p.720-723, 1990. 